\title{
A Novel Loss-of-Function Mutation in HOXB1 Associated with Autosomal Recessive Hereditary Congenital Facial Palsy in a Large Iranian Family
}

\author{
Mohammad Yahya Vahidi Mehrjardi ${ }^{a}$ Reza Maroofian ${ }^{d}$ Seyed M. Kalantar ${ }^{b}$

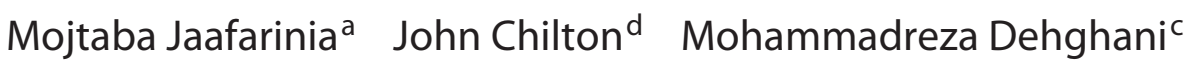 \\ a Department of Genetics, Marvdasht Branch, Azad University, Marvdasht, and ${ }^{b}$ Reproductive and Genetic Unit, \\ Yazd Research and Clinical Center for Infertility, and ' Medical Genetics Research Center, Shahid Sadoughi University \\ of Medical Sciences, Yazd, Iran; 'Wellcome Wolfson Medical Research Centre, University of Exeter Medical School, \\ Exeter, UK
}

\section{Keywords}

Autosomal recessive $\cdot$ Congenital facial palsy $\cdot$ HOXB1 . Moebius syndrome

\begin{abstract}
Hereditary congenital facial palsy (HCFP) is a rare congenital cranial dysinnervation disorder, recognisable by non-progressive isolated facial nerve palsy (cranial nerve VII). It is caused by developmental abnormalities of the facial nerve nucleus and its nerve. So far, 4 homozygous mutations have been identified in 5 unrelated families (12 patients) with HCFP worldwide. In this study, a large Iranian consanguineous kindred with 5 members affected by HCFP underwent thorough clinical and genetic evaluation. The candidate gene HOXB1 was screened and analysed by Sanger sequencing. As in previous cases, the most remarkable findings in the affected members of the family were mask-like faces, bilateral facial palsy with variable sensorineural hearing loss, and some dysmorphic features. Direct sequencing of the candidate gene HOXB1 identified a novel homozygous frameshift mutation (c.296_302del; p.Y99Wfs*20) which cosegregated with the disease phenotype within the extended family. Our findings expand the mutational spectrum of HOXB1 involved in HCFP and consolidate the role of the gene
\end{abstract}

\section{KARGER}

(C) 2017 S. Karger AG, Basel

E-Mail karger@karger.com

www.karger.com/msy in the development of autosomal recessive HCFP. Moreover, the truncating mutation identified in this family leads to a broadly similar presentation and severity observed in previous patients with nonsense and missense mutations. This study characterises and defines the phenotypic features of this rare syndrome in a larger family than has previously been reported.

(c) 2017 S. Karger AG, Basel

Hereditary congenital facial palsy (HCFP) is a rare condition belonging to the family of congenital cranial dysinnervation disorders (CCDD) and is characterised by the paralysis or weakness of facial muscles due to underdevelopment of the facial branchiomotor nucleus and its nerves (particularly the VI and VII cranial nerves) [Terzis and Anesti, 2011; Gutowski and Chilton, 2015; Chilton and Guthrie, 2016]. Cranial nerve dysfunction often presents at birth with multiple problems, mainly due to the functional and aesthetic consequences such as inadequate sucking and feeding as well as lack of facial expression [Ciorba et al., 2015]. Although HCFP and Moebius syndrome (MBS) share some clinical features, they are different clinical entities with a different pathogenesis [MacKinnon et al., 2014]. MBS (MIM 157900) is

Mohammadreza Dehghani

Medical Genetics Research Center, Shahid Sadoughi University of Medical Sciences Shahid Ghandi Blvd.

Safaieh, Yazd 8915887857 (Iran)

E-Mail reza.dehghani@unipv.it 
usually sporadic and associated with the involvement of other cranial nerves, commonly the abducens nerve in addition to orofacial and limb malformations as well as defects of the musculoskeletal system [MacKinnon et al., 2014]. De novo mutations in 2 genes, PLXND1 and $R E V 3 L$, were recently found to be associated with MBS [Tomas-Roca et al., 2015]. Congenital facial palsy (CFP) is classified as acquired or developmental, unilateral or bilateral, and complete (palsy) or incomplete (paresis). A careful diagnostic work-up and differential diagnosis are important in order to determine the aetiology, as the prognosis and the therapeutic approach differs, depending on the underlying pathophysiology [Terzis and Anesti, 2011; Ciorba et al., 2015]. Based on previous studies, genetic heterogeneity and different modes of inheritance have been suggested for this disorder. Two genetic loci, termed HCFP1 (MIM \%601471) and HCFP2 (MIM \%604185), have been defined in 2 families with autosomal dominant HCFP, but neither gene has been identified [Kremer et al., 1996; Verzijl et al., 1999; Michielse et al., 2006]. The only known autosomal recessive HCFP (HCFP3) is caused by biallelic mutations in HOXB1 (MIM \%142968) in chr17q21.32 (MIM 614744). Recently, 4 mutations (3 missense and 1 nonsense) in the HOXB1 gene have been identified in 12 patients from 5 unrelated consanguineous families with HCFP3 (Fig. 1) [Webb et al., 2012; Uyguner et al., 2015; Vogel et al., 2016; Sahin et al., 2017]. HOXB1 is a homeobox transcription factor which is part of a developmental regulatory system expressed in the embryological spinal cord and hindbrain and is essential for rhombomere 4 (r4) development [Webb et al., 2012].

This study presents an Iranian extended consanguineous family in which 5 members suffer from HCFP. Genetic screening of the only currently known candidate gene for the condition revealed a novel homozygous truncating mutation in $H O X B 1$.

\section{Patients and Methods}

The patient medical records were reviewed and clinical examinations including opthalmological and auditory exams were performed. A detailed family history was collected and a pedigree was drawn. Blood samples from the 5 affected members of the family as well as their parents and unaffected siblings were obtained for genetic testing.

Genetic Study

Genomic DNA was extracted from blood samples using Qiagen Gentra Puregene DNA isolation kits (Qiagen, Valencia, CA, USA). Mutation analysis was performed by PCR amplifying all exons and exon-intron boundaries of the $H O X B 1$ gene, using 2 pairs of primers. All the resulting amplicons were Sanger sequenced. Sequencing results from affected and unaffected individuals were analysed using FinchTV Software. Primer sequences and screening conditions are available upon request.

\section{Results}

We investigated a large Iranian consanguineous kindred including 5 affected individuals (Fig. 1A; V-2, 2 years old; V-5, 9 years old; V-7, 11 years old; VI-3, 24 years old, and VI-18, 25 years old) who were initially suspected to have MBS. The initial clinical presentation was congenital non-progressive mask-like faces and bilateral facial palsy. Due to facial muscle weakness and paresis, these individuals could not smile, frown, or cry. They had severe feeding difficulty and inadequate suckling of milk during infancy; however, they could discriminate tastes. Salivation and lacrimation were both intact. Two patients also had congenital mixed, sensorineural and conductive bilateral hearing loss and were using hearing aids. Their hearing impairment was non-progressive and moderate to severe. Additional clinical features of the patients were facial dysmorphism, including a smooth philtrum, lowset ears, external ear malformation (in 3 patients), midface retrusion, upturned nasal tip, and tooth abnormalities (Fig. 1C). Brain CT scans performed on 2 affected individuals did not identify any abnormality. The karyotype of one patient was examined and found to be normal. All 5 affected individuals showed typical signs of HCFP with full eye motility and no eye movement abnormalities. Based on the extended 3-generation pedigree, with the presence of consanguinity and healthy parents, an autosomal recessive mode of inheritance was suggested for the disease.

The disease phenotype and family history was not consistent with a diagnosis of MBS; we therefore screened the only known autosomal recessive HCFP-causing gene HOXB1. Direct sequencing of the HOXB1 gene identified a novel 7-bp deletion - (NM_002144.3, hg19) chr17: g.46607965_46607971delAGAGGGT; c.296_302del; Y99Wfs*20 - present in a homozygote state in the first coding exon (Fig. 1B). The mutation resulted in a frameshift predicted to cause premature termination of the protein. This variant has not been reported in a heterozygous or homozygous state in publicly available databases, including dbSNP (build 138), the Exome Sequencing Project databases (http://evs.gs.washington.edu/ EVS/), gnomeAD browser (http://gnomad.broadinsti-
262

Mol Syndromol 2017;8:261-265

DOI: $10.1159 / 000477752$
Vahidi Mehrjardi et al. 


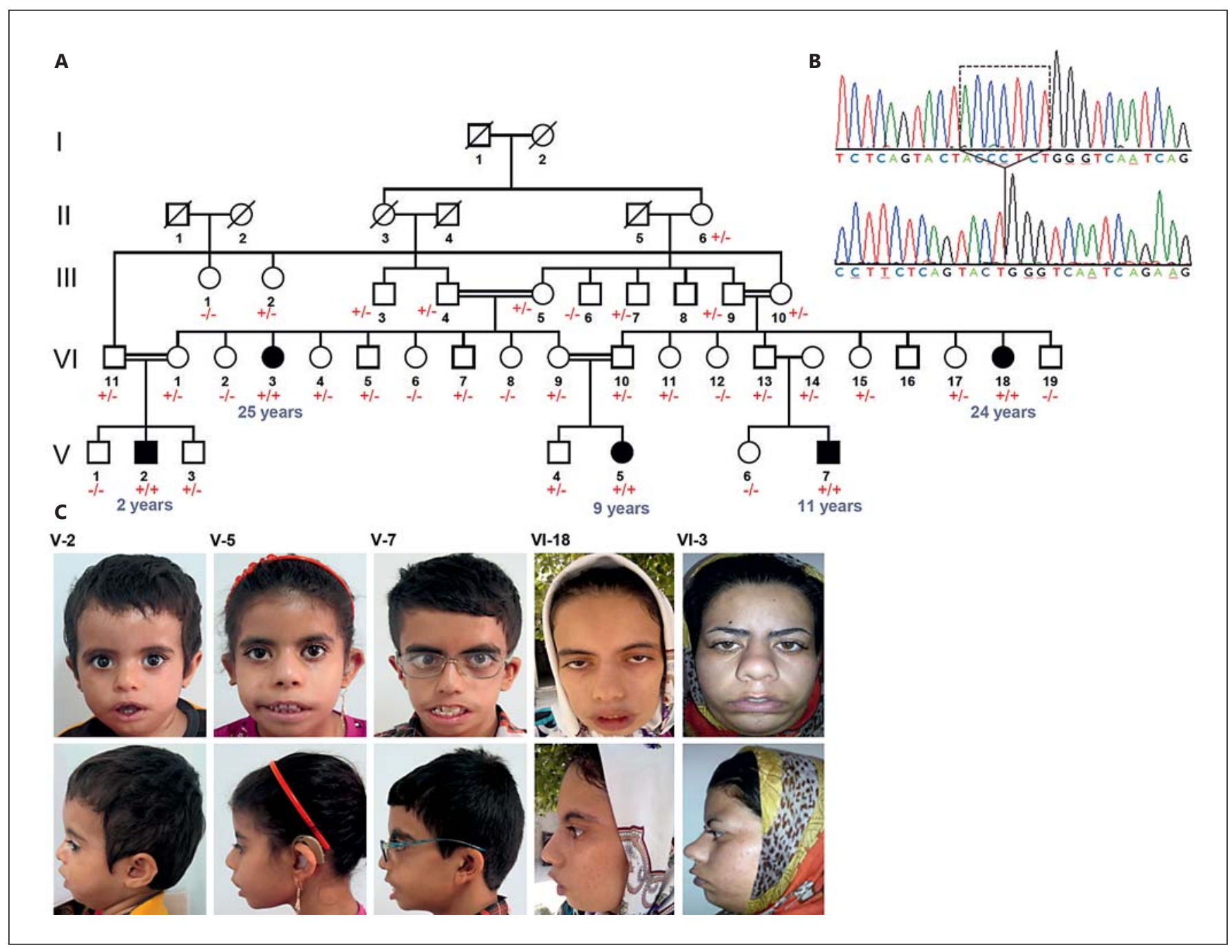

Fig. 1. A Pedigree of the Iranian family with 5 individuals affected by hereditary congenital facial palsy (indicated as solid black symbols). Segregation of the loss-of-function mutation in the family with the mutant allele is shown by + and the wild-type allele is shown by - . The ages of the affected individuals are shown below the symbols. B A section of Sanger sequencing chromatograms for the homozygous mutation (bottom) and wild type (top) are depicted and demonstrate the deletion (box). C Pictures of the patients corresponding to the pedigree. tute.org), the 1000 Genome Project (http://1000genomes. org/), The Greater Middle East Variome project (http:// igm.ucsd.edu/gme/index.php) or our in-house database of 500 Iranian Whole Exome Sequencing. The mutation completely co-segregates with the disease phenotype in this large family (Fig. 1A). Clinical features of the affected family members are summarised in Table 1 and compared to previously reported patients with $H O X B 1$ mutations.
Autosomal Recessive Hereditary Congenital Facial Palsy

\section{Discussion}

Until now, there are 17 HCFP patients reported worldwide, including 5 patients from this study, in whom causative mutations have been identified (Fig. 2) [Webb et al., 2012; Uyguner et al., 2015; Vogel et al., 2016; Sahin et al., 2017]. Our findings consolidate the role of HOXB1 in autosomal recessive HCFP and expand the mutational and phenotypic spectrum of $H O X B 1$ variations in causing CCDD. The mutation we report here would likely result in a null mutation due to nonsense-mediated decay of the

Mol Syndromol 2017;8:261-265 DOI: $10.1159 / 000477752$ 


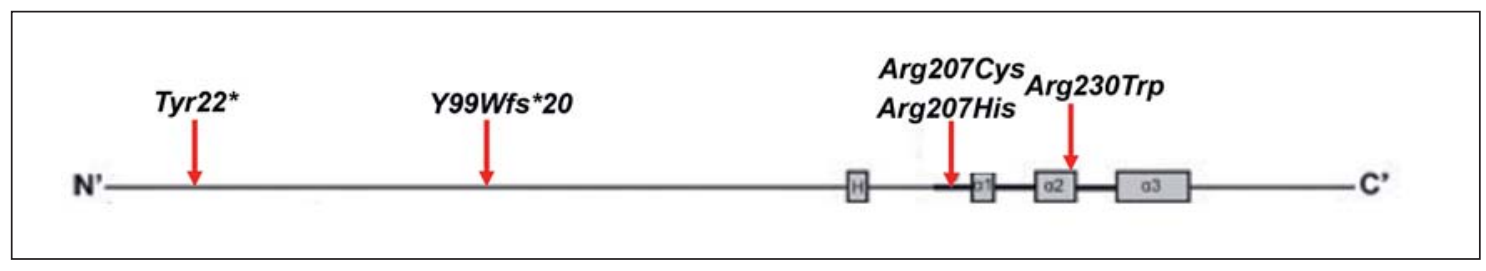

Fig. 2. A schematic representation of $H O X B 10$ homozygous mutations identified so far indicated by the red arrows.

Table 1. Clinical features of affected members of the Iranian family with HCFP and previously reported cases

\begin{tabular}{|c|c|c|c|c|c|}
\hline Features & Present family & Sahin et al., 2017 & Vogel et al., 2016 & Uyguner et al., 2015 & Webb et al., 2012 \\
\hline \multicolumn{6}{|l|}{ Molecular findings } \\
\hline NM_002144.3 & c.296_302del & c. $688 \mathrm{C}>\mathrm{T}$ & c. $66 \mathrm{C}>\mathrm{G}$ & c. $620 \mathrm{G}>\mathrm{A}$ & c. $619 \mathrm{C}>\mathrm{T}$ \\
\hline NP_002135.2 & p.Y99Wfs*20 & p.Arg230Trp & p.Tyr22* & p.Arg207His & p.Arg207Cys \\
\hline Localization & exon 1 & exon 2 & exon 1 & exon 2 & exon 2 \\
\hline Protein domain & - & homeodomain & $\mathrm{N}$ terminal arm & homeodomain & homeodomain \\
\hline Origin & Iranian & Turkish & Moroccan & Turkish & $\begin{array}{l}\text { German } \\
\text { American }\end{array}$ \\
\hline Gender & $\mathrm{F}(2), \mathrm{M}(3)$ & $\mathrm{F}(2), \mathrm{M}(1)$ & $\mathrm{F}(1), \mathrm{M}(3)$ & $\mathrm{F}(1)$ & $\mathrm{F}(1), \mathrm{M}(3)$ \\
\hline Consanguinity & $5 / 5$ & $2 / 3$ & $4 / 4$ & $0 / 1$ & $2 / 4$ \\
\hline \multicolumn{6}{|l|}{ Craniofacial findings } \\
\hline Masked facies & $5 / 5$ & $3 / 3$ & $4 / 4$ & $1 / 1$ & $4 / 4$ \\
\hline Bilateral facial weakness & $5 / 5$ & $3 / 3$ & $4 / 4$ & $1 / 1$ & $4 / 4$ \\
\hline Full oculomotor motility & $5 / 5$ & $4 / 4$ & $4 / 4$ & $1 / 1$ & $4 / 4$ \\
\hline Esotropia/esophoria & $1 / 5$, right & $0 / 3$ & $1 / 4$ & $1 / 1$, left & $4 / 4$ \\
\hline Ptosis & $1 / 5$, bilateral & $0 / 3$ & $0 / 4$ & $1 / 1$, left & $0 / 4$ \\
\hline Smooth philtrum & $2 / 5$ & $3 / 3$ & $3 / 4$ & $0 / 1$ & $2 / 4$ \\
\hline Midface retrusion & $4 / 5$ & $3 / 3$ & $4 / 4$ & $1 / 1$ & $4 / 4$ \\
\hline Upturned nasal tip & $1 / 5$ & $3 / 3$ & $4 / 4$ & $1 / 1$ & $4 / 4$ \\
\hline Low-set ears & $1 / 5$ & $0 / 3$ & $1 / 4$ & $1 / 1$ & $3 / 4$ \\
\hline Micrognathia & $0 / 5$ & $0 / 3$ & $0 / 4$ & $0 / 1$ & $2 / 4$ \\
\hline External ear malformations & $3 / 5$ & $0 / 3$ & $4 / 4$ & $0 / 1$ & $0 / 4$ \\
\hline \multicolumn{6}{|l|}{ Other findings } \\
\hline Speech delay & $0 / 5$ & $2 / 3$ & $4 / 4$ & $0 / 1$ & $2 / 4$ \\
\hline Feeding difficulties & $5 / 5$ & $3 / 3$ & $4 / 4$ & $0 / 1$ & $2 / 4$ \\
\hline Sensorineural hearing loss & $2 / 5$ & $3 / 3$ & $2 / 4$ & $0 / 1$ & $4 / 4$ \\
\hline $\begin{array}{l}\text { Abnormal cochlear } \\
\text { function }\end{array}$ & $2 / 5$ & $3 / 3$ & $2 / 4$ & $0 / 1$ & $2 / 4$ \\
\hline Cochlear structural defect & $1 / 5$ & n.d. & $0 / 4$ & n.d. & $1 / 4$ \\
\hline
\end{tabular}

F, female; HCFP, hereditary congenital facial palsy; M, male; n.d., not determined.

HOXB1 transcript. A previous report drew parallels between the Arg209Cys point mutation and the HoxB1 $1^{-/-}$ mouse [Webb et al., 2012]. Our data strongly support the conclusion that the key overlapping clinical features - facial weakness and dysmorphia - result from the loss of HOXB1 function.

Understanding the association of CFP with concomitant esotropia is important in achieving a differential diagnosis of HCFP and MBS. HOXB1 mutations have not been found in patients with classic MBS in this study or previous reports, suggesting that it may be a useful diagnostic marker [Ciorba et al., 2015]. Our data also demonstrate that further analysis of the regulation and function of $H O X B 1$ will help elucidate CCDD pathology.

The loss-of-function mutation in 5 affected members of the current report exhibits a similar clinical presentation to that observed in previous patients with HOXB1 mutations. Moreover, there is no clear genotype-phenotype correlation with regards to the type of mutations, their location in the protein, and severity of the pheno- 
type. However, as seen in Moroccan patients with a lossof-function mutation, 3 of these Iranian patients showed external ear malformations, which were not observed in the other 8 reported patients with missense mutations or in the HOXB1-deficient mouse model [Webb et al., 2012; Uyguner et al., 2015; Vogel et al., 2016; Sahin et al., 2017]. Further cases of HOXB1-related HCFP would be necessary before we can confidently establish an association between the genotype and the phenotype of external ear malformation in this rare disease. External ear malformation is not an uncommon occurrence in MBS patients [Suvarna et al., 2006]. Additionally, a combined phenotype of CFP and external ear anomalies has been reported in an unknown autosomal dominant syndrome characterised by conductive deafness due to stapedial anomalies, external ear malformations, and CFP [Sellars and Beighton, 1983].

We identified a novel homozygous truncating mutation in HOXB1 in a consanguineous kindred with HCFP, which is broadly similar to the clinical phenotype of patients who were previously described. The mutation identified in this family is the first indel described in HOXB1 so far. It must be emphasised that in individuals with CFP, hearing loss, dysmorphic facial features, and full eye motility, HOXB1 mutation screening should be considered to help with an accurate diagnosis.

\section{Acknowledgments}

We would like to thank all the participating patients and their families for their support in this ongoing research.

\section{Statement of Ethics}

The participating family members and their parents provided written informed consent. This study was approved by the local ethical committee of the Shahid Sadoughi University of Medical Sciences in Yazd, Iran.

\section{Disclosure Statement}

The authors have no conflicts of interest to disclose.

\section{References}

Chilton JK, Guthrie S: Axons get ahead: insights into axon guidance and congenital cranial dysinnervation disorders. Dev Neurobiol 2016, E-pub ahead of print.

Ciorba A, Corazzi V, Conz V, Bianchini C, Aimoni C: Facial nerve paralysis in children. World J Clin Cases 3:973-979 (2015).

Gutowski NJ, Chilton JK: The congenital cranial dysinnervation disorders. Arch Dis Child 100:678-681 (2015).

Kremer H, Kuyt LP, van den Helm B, van Reen $\mathrm{M}$, Leunissen JA, et al: Localization of a gene for Möbius syndrome to chromosome $3 \mathrm{q}$ by linkage analysis in a Dutch family. Hum Mol Genet 5:1367-1371 (1996).

MacKinnon S, Oystreck DT, Andrews C, Chan WM, Hunter DG, Engle EC: Diagnostic distinctions and genetic analysis of patients diagnosed with moebius syndrome. Ophthalmology 121:1461-1468 (2014).

Michielse CB, Bhat M, Brady A, Jafrid H, van den Hurk JA, et al: Refinement of the locus for hereditary congenital facial palsy on chromosome 3q21 in two unrelated families and screening of positional candidate genes. Eur J Hum Genet 14:1306-1312 (2006).
Sahin Y, Güngör O, Ayaz A, Güngör G, Sahin B, et al: A novel homozygous HOXB1 mutation in a Turkish family with hereditary congenital facial paresis. Brain Dev 39:166-170 (2017).

Sellars S, Beighton P: Autosomal dominant inheritance of conductive deafness due to stapedial anomalies, external ear malformations and congenital facial palsy. Clin Genet 23:376379 (1983).

Suvarna J, Bagnawar M, Deshmukh CT: Moebius syndrome with total anomalous pulmonary venous connection. Indian J Pediatr 73:427429 (2006).

Terzis JK, Anesti K: Developmental facial paralysis: a review. J Plast Reconstr Aesthet Surg 64: 1318-1333 (2011).

Tomas-Roca L, Tsaalbi-Shtylik A, Jansen JG, Singh MK, Epstein JA, et al: De novo mutations in PLXND1 and REV3L cause Möbius syndrome. Nat Commun 6:7199 (2015).
Uyguner ZO, Toksoy G, Altunoglu U, Ozgur H, Basaran S, Kayserili H: A new hereditary congenital facial palsy case supports arg 5 in HOX-DNA binding domain as possible hot spot for mutations. Eur J Med Genet 58:358363 (2015).

Verzijl HT, van den Helm B, Veldman B, Hamel BC, Kuyt LP, et al: A second gene for autosomal dominant Möbius syndrome is localized to chromosome 10q, in a Dutch family. Am J Hum Genet 65:752-756 (1999).

Vogel M, Velleuer E, Schmidt-Jiménez LF, Mayatepek E, Borkhardt A, et al: Homozygous HOXB1 loss-of-function mutation in a large family with hereditary congenital facial paresis. Am J Med Genet A 170:1813-1819 (2016).

Webb BD, Shaaban S, Gaspar H, Cunha LF, Schubert CR, et al: HOXB1 founder mutation in humans recapitulates the phenotype of $\mathrm{Hoxb1}^{-/-}$mice. Am J Hum Genet 91:171-179 (2012). 